Article

\title{
Social-Aware Relay Selection for Cooperative Multicast Device-to-Device Communications
}

\author{
Francesco Chiti (D), Romano Fantacci (1D)and Laura Pierucci * \\ Department of Information Engineering, University of Florence, 50139 Florence, Italy; \\ francesco.chiti@unifi.it (F.C.); romano.fantacci@unifi.it (R.F.) \\ * Correspondence: laura.pierucci@unifi.it; Tel.: +39-055-275-8626
}

Received: 29 September 2017; Accepted: 28 November 2017; Published: 4 December 2017

\begin{abstract}
The increasing use of social networks such as Facebook, Twitter, and Instagram to share photos, video streaming, and music among friends has generated a huge increase in the amount of data traffic over wireless networks. This social behavior has triggered new communication paradigms such as device-to-device (D2D) and relaying communication schemes, which are both considered as strong drivers for the next fifth-generation (5G) cellular systems. Recently, the social-aware layer and its relationship to and influence on the physical communications layer have gained great attention as emerging focus points. We focus here on the case of relaying communications to pursue the multicast data dissemination to a group of users forming a social community through a relay node, according to the extension of the D2D mode to the case of device-to-many devices. Moreover, in our case, the source selects the device to act as the relay among different users of the multicast group by taking into account both the propagation link conditions and the relay social-trust level with the constraint of minimizing the end-to-end content delivery delay. An optimization procedure is also proposed in order to achieve the best performance. Finally, numerical results are provided to highlight the advantages of considering the impact of social level on the end-to-end delivery delay in the integrated social-physical network in comparison with the classical relay-assisted multicast communications for which the relay social-trust level is not considered.
\end{abstract}

Keywords: multicast; device-to-device communications; Internet of Things; mobile social networks

\section{Introduction}

In the incoming fifth-generation (5G) system, device to device (D2D) and relaying communications are envisaged as enablers to face the huge amount of data traffic due to novel and advanced applications and services. In particular, the emerging trend of sharing photos, video, and music among friends requires a large amount of data at a high data rate. In D2D communications, the devices can share the same relevant contents or help the neighbours to deliver data by establishing a direct link without (or with limited) involvement of a base station (BS) or eNodeB. As the D2D communications occur over short distances, they can support a higher data rate with respect to infrastructured communications. Furthermore, D2D communications enhance the spectral efficiency, lighten areas with elevate traffic and improve the user quality of experience.

In the foreseen integrated $5 \mathrm{G}$ and Internet of Things (IoT) infrastructures, short-range D2D communications can interconnect heterogeneous devices with lower-energy consumptions guaranteeing proximity services for $5 \mathrm{G} / \mathrm{IoT}$ networks. The D2D concept allows for a direct connection between D2D pairs of devices, considerably (or fully) reducing the exchange of traffic requests with the BS.

The D2D approach is also useful in the multicast context, where multiple cellular/IoT devices have to receive the same data from the BS. Various devices can self-organize into clusters, and some of 
these can be selected to act as a relay to help the forwarding of data, particularly to the end-nodes of the networks and the offloading of traffic from the BS.

Relay-assisted communications have been actively studied and are already considered in the standardization process of mobile broadband communication systems, such as in the Third Generation Partnership Program (3GPP) Long-Term Evolution Advanced (LTE-Advanced), IEEE 802.16j and the IEEE $802.16 \mathrm{~m} \mathrm{[1,2]} \mathrm{to} \mathrm{improve} \mathrm{the} \mathrm{cell-edge} \mathrm{coverage} \mathrm{radius} \mathrm{and} \mathrm{to} \mathrm{provide} \mathrm{high} \mathrm{data} \mathrm{rates} \mathrm{to} \mathrm{the} \mathrm{users}$ located in the cell-edge or in coverage holes.

In addition, cooperative multicast relaying can improve data rates as a result of the shorter distance from multicast devices with respect to the direct transmission, for which the worst propagation channel limits the available transmission rate. In the next generation, 5G networks, cognitive relaying and cooperative D2D relaying will continue to be the main players.

In cooperative $\mathrm{D} 2 \mathrm{D}$ communications, the common concept is that all the devices can relay to each other, but, for example, the battery charging can often limit data forwarding assistance because of the energy consumption. If D2D communication is under a partial operator control, the user acting as a relay can have a bill reduction, or the user can offer his battery and bandwidth consumption only if he wants to help his friend.

Recently, the increasing demand for social applications, such as Facebook, Twitter, YouTube, Instagram, and so on, has suggested integrating the social behavior on a cooperative D2D/relaying design, in which the D2D users can communicate directly with each other and can exchange content mainly if they are friends. Different social communities, in which each participant has the same interest in content, can be formed, for example, by tracking friends, kin and colleagues that share content frequently by online social networks. Social media networking indeed represents a disruptive paradigm leading the transition to the "Web2.0". The proliferation of popular applications for smartphones points out a constant trend for geo-referenced services with an increased level of integration among different communities, thus making the content dissemination more pervasive and instantaneous.

Generally, the social trust model for cooperative D2D communications is built by two different layers that interact each other:

- The online social network layer, which indicates the different levels of relationships among the D2D users.

- The offline mobile communications network layer, which determines the wireless connections subject to the channel propagation conditions.

For example, a high level of relationships can exist between two users of the social layer because they belong to the same group, such as family or colleagues, but the same connection cannot exist in the physical layer because there is no proximity or there are bad propagation conditions of the link, as shown in Figure 1.

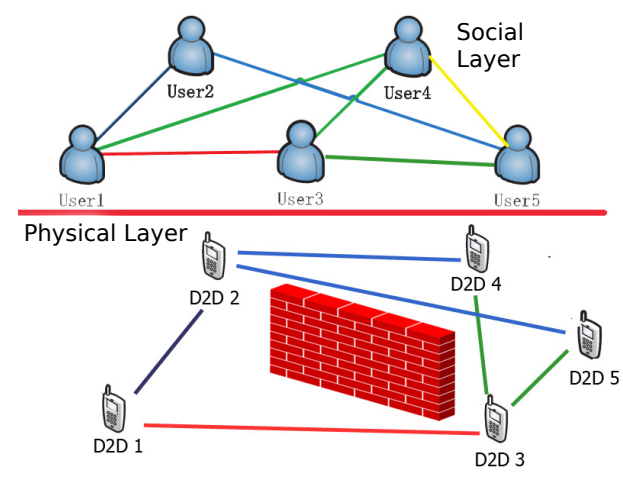

Figure 1. Social-physical layers for cooperative device-to-device (D2D) relaying. 
The social-trust level can be evaluated through the analysis of the contents and account information shared, for example, by tracking online social network services and calculating the ranking of the user to spread the content (this is related to the number of friends). The social relationships can be retrieved by matching mobile phones' contact books: if two users are colleagues or members of the same family, it is very likely that they have many phone contacts in common.

Moreover, when the D2D relay has received popular content from the BS, it can share this to other users in proximity through D2D communications, and a new entry in the group of friends can make available this content, considerably decreasing the traffic load of the BS. The geographical location can impact this social transfer of the same data; for example, in a school building, it is highly likely that different students download the same viral video, and thus a proximity area can be a characteristic for content delivery.

Current researches analyze the cooperative communication gain for proper relay selection by considering a weighted tradeoff between the social trust information and the physical constraints in order to maximize the throughput with respect to the direct transmission, mainly for scenarios with only one destination.

This paper presents a multicast scenario with a source (either the eNodeB or a D2D user), which transmits the same data content to many destinations through the selection of the best relay among different alternatives, by taking into account the relay social-trust level and propagation condition links to optimize the end-to-end delivery delay. This is defined as the time required to deliver data from the source through the relay to all the destinations belonging to the multicast group (i.e., the social community). The relay itself is interested in receiving data content because it can belong to a multicast group. The social-trust level is related to the social relationships of the relay with the source and represents the part of the transmit power that the relay is available to give as friend to forward the data to the multicast community and as a consequence, the availability to consume its own battery. The performance of this social cooperative multicast system is analyzed by considering that the direct link among the source and destinations is not available because of path loss and shadowing effects and that all the D2D multicast devices have to receive all the data.

The remainder of the paper is organized as follows. Section 2 provides a literature survey. In Section 3, the system model is introduced, and in Section 4, we provide the simulation results for the end-to-end delivery time performance evaluation of the integrated social multicast D2D-based system. Finally, the conclusions are drawn.

\section{Literature Review}

D2D communications are foreseen to be of paramount importance in $5 \mathrm{G}$ systems for improving system capacity and for offloading traffic from a BS. In this context, devices can autonomously establish direct connections sharing the spectrum of cellular systems (underlaid D2D communications) and resource allocation, and management approaches have to be handled to provide proximity services [3,4]. Different research proposes interference-avoiding schemes under the management of network infrastructure to prevent harmful interference among D2D and cellular users or to analyze autonomous D2D data transmissions with guaranteed limited and tolerable interference on cellular users. Others challenges faced regard the significant reduction of traffic load and communication delay [5] and the neighbor discovery methods to detect proximity users in cellular networks with underlaid D2D communications [6].

As users can self-organize with direct connections, D2D cooperation can be the main means to improve throughput and energy efficiency. Unfortunately, when D2D users cooperate, the devices acting as relays expend extraordinary energy for data transmissions, even over short distances. Hence, it is necessary to select D2D relays among multiple devices by considering whether a device has already been selected as a relay many times or if it has to handle too many cooperative users to avoid excessive energy consumption of the relay and to decrease the life-time of the system [7]. 
In D2D communications networks, the mobile devices are intermittently connected in an ad hoc manner, and the topology of the network can be highly dynamic. In [8], a novel opportunistic network routing protocol based on social rank and intermeeting time is considered. Cooperative multicast transmissions, for which different devices with good channel conditions are selected as relay nodes, improve the achievable data rate with respect to the direct transmission from the BS, which suffers from the constraint of the worst propagation channel as a result of the long distance, for example. In the literature, various relaying technologies are considered, such as decode and-forward (DF) and amplify-and-forward (AM) relaying. Different relaying strategies have been proposed, such as relay selection, in which multiple relay nodes allow for a more efficient use of the system resources through the selection of the best source-relay and relay-destination channel, and incremental relaying, in which feedback from the destination about the success or failure of direct source-destination transmission is used. Full duplex (FD) relays offer high spectral efficiency but suffer from strong self-interference and loop interference if multiple antennas are installed on each relay. On the other hand, half-duplex (HD) relays, for which the source transmits information to relays in the broadcast phase and then relays this forward to destinations in the successive time slots, causes multiplexing loss. Many methods have recently been proposed to overcome the multiplexing loss in HD relaying. Successive relay techniques [9] are analyzed to improve the spectral efficiency of HD relays, in which a pair of relays is selected, and while one relay receives data from the source, the other transmits the previously received data to the destination. The use of a buffer at the relay nodes, "buffer-aided relaying methods", as in [10-15], increases the spectral efficiency with respect to HD relaying without a buffer. This approach allows for selecting the best HD buffer-aided relay among the various relay-destination links (opportunistic relaying schemes), to transmit the data if the channel conditions are good or buffer it otherwise, as in max-max relay selection by Ikhlef et al. [16] or the max-link relay selection scheme by Krikidis et al. [17].

Recently, several analyses of user behaviors and their social relationships are addressed by considering the most popular content shared on social networks such as Facebook, Instagram, YouTube, and WhatsApp, as well what the influences of other friends, media, bloggers and advertising are. Different probabilistic models are proposed to predict the rate of downloading and the requests' evolution of some content.

The new vision is to join the social relationships information and proximity-based communications to build an autonomous, trustworthy network of smart IoT devices. The impact of social relationships on the overall throughput of a D2D communication system is considered in [18] with the stop-wait approach for relay selection. The distributed resource allocation, mainly based on cooperative game theory to exploit diverse social relationships on a physical domain, is analyzed in [19]; a Bayesian model for social relationships and a coalitional graph game for efficient data distribution is proposed in [20]; the social characteristics are used to help ad hoc peer discovery in [21]; a neighbor discovery method and a dynamic detection of overlapping social communities is highlighted in [6]. Zhang et al. [22] propose a model for the delivery of content in the online social level jointly with the optimization of a traffic offloading process for the D2D communications layer. In [23], the sociality among IoT devices is used to model the trustworthiness for successful D2D-based content delivery to significantly reduce the impact of malicious behavior.

In [18], the optimal stopping policy for relay selection is proposed for the case of cooperative D2D relaying, but the multicast context is not considered. This policy, suitably elaborated for the multicast case, is considered as the benchmark for our throughput performance. In [24], a cooperative multicast scheme with underlaid D2D communications is proposed, in which social relationships drive the relay selection. The selected relay nodes receive broadcast messages from the BS on downlink (DL) band and then forward these to multicast users in the uplink (UL) band via D2D communications to increase the multicast transmission rate.

In the proposed paper, a cooperative multicast scenario is also considered as in [24] with the difference that the best relay is selected to optimize the global end-to-end delivery delay metric. 
Moreover, the same content broadcast from the BS to the relay is distributed to the users of the multicast group by assuming that one message is delivered only when all the users in the multicast group have received it. We would like to stress that our focus is on a social-physical scenario, for which, in particular, the social level trust is considered to have a more general meaning with respect to classical real-world networks. In particular, the social level trust of a device is related to its need or interest in receiving a given information flow, for example, according to the novel paradigm of Fog computing/networking and applications in which clusters of smart devices collaborate toward a common goal. As a consequence, the end-to-end delivery delay is considered as the most important parameter to evaluate the optimal social-aware relay selection mechanism in the case of multicast transmission. Therefore, in this paper, the best relay is selected among the different D2D devices according to the sociality index, the proximity distance to the multicast users and the links' propagation conditions.

\section{System Model}

We focus on a cooperative multicast context, in which end users receive the requested content not directly from the BS but via other users acting as a relay in D2D communications.

In the considered social-physical scenario, the users can communicate directly with other multicast users and exchange shared content mainly if they are friends and according to the quality of the D2D connection links. In particular, we refer here to the classical social-physical architecture, which entails two layers, as in Figure 1. In this context, the source (either the eNodeB or a D2D node) can deliver the same content to a group of D2D devices in a small area, and as the node density increases, various devices can act as relays to forward multicast traffic to the end-devices of the social communities and vice versa. The devices selected as relays may also be interested in receiving the same data content because they could belong to the multicast group. According to the multicast concept, data is delivered if all the destinations have received it. Moreover, we assume that the D2D multicast group can be very far from the BS and that the direct path cannot exist or suffers from a deep attenuation due to path-loss and shadowing effects.

A device can leave or join the multicast community according to his mobility. However, we assume that the D2D devices remain in the same location during a D2D communication transmission period (e.g., in the order of milliseconds), while their positions can change across different periods because of users' mobility. The D2D social-trust levels and the forwarding metric related to the channel quality are known at the source and D2D/relays.

We consider a relay-assisted network in which a source $S$ transmits to many destinations $D_{i}$ with $i=1 \ldots . M$ through different relays $R_{j}$ with $j=1, \ldots N$, as in Figure 2 . We assume flat block fading on all the links, such that the channel coefficients can be assumed to be constant during one time transmission period and can change from one period to the next. One relay is selected among $N$ to serve the multiple destinations according to the best joint quality of the social and physical layers. The relay nodes use HD decoding and the forward relaying mode. The nodes can retrieve their social relationships information, for example, from the BS, reporting the analysis of content sharing or account information by accessing online social websites such as Facebook, Twitter, YouTube, Instagram, and so forth. These social trust values tend to be stable over the time reporting friendships and acquaintances among users. Moreover, for example, Twitter associates with each tweet the exact location (latitude and longitude), and this might allow the nodes the possibility to know their related positions and also discover their neighbors in mobility. 


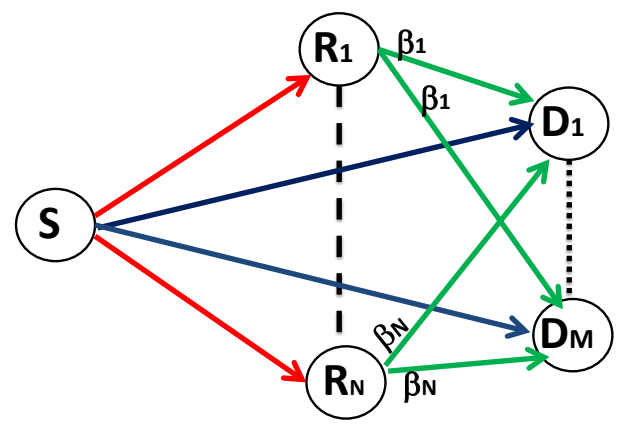

Figure 2. Multicast model based on social-trust level.

As in [18], the social trust impacts the transmission power of the relay node: if a stronger social-trust relationship exists between the source and relay, the relay provides more power resources to help the source node in retransmission.

Therefore, the selected relay $R_{j}$ transmits to the multiple destinations with a power proportional to the strength of the social-trust value $\beta_{j}$. For each relay-destination link, the $\gamma$ signal-to-noise ratio (SNR) at the destination $D_{i}$ is related to the path-loss, the Rayleigh flat fading, and the zero-mean additive white Gaussian noise (AWGN) with variance $\sigma_{n}^{2}$, and it can be expressed as

$$
\gamma_{R_{j}, D_{i}}=\frac{P_{R_{j}}\left|h_{R_{j}, D_{i}}\right|^{2}}{\sigma_{n}^{2} d_{R_{j}, D_{j}}^{\alpha}}
$$

where $P_{R_{j}}$ is the transmit power of the $j$ th relay, $d_{R_{j}, D_{i}}$ stands for the distance of relay $R_{j}$ from destinations $D_{i}, \alpha$ is the path-loss coefficient, and $h_{R_{j}, D_{i}}$ are the Rayleigh fading coefficients.

In the multicast transmission mode, minimizing the delivery latency necessary to receive the data to all the destinations is the main objective.

As a consequence, the aim of this paper is to minimize the end-to-end delivery delay that is obtained considering $T_{j}$, the time needed to transmit from the source to the $j$ th selected relay, and $T_{j, I}$, the overall time for transmission from the relay to the destinations. In the case of multicast transmission, the time to deliver the data from the relay to destinations is related not only to the link with the higher delay, because all the destinations have to receive the same content, but also to the social-trust level of the relay, which allows or does not allow the transmission to destinations. The quality of physical channels is directly related to the SNR and consequently to the data rate available, while the social trust $\beta_{j}$ assures the social link among the nodes. These two aspects, the social relationship and the channel quality, must to be balanced to optimize the end-to-end delivery delay for the cooperative multicast D2D relaying.

Accordingly to this, the channel rate $C_{j}$ for the link between the source and the $j$ th relay is

$$
C_{j}=W \log _{2}\left(1+\gamma_{S, R_{j}}\right)
$$

where $W$ is the system bandwidth and $\gamma_{S, R_{j}}$ is the SNR of the link between the source and the $j$ th relay; the multicast channel rate can be expressed for each link from the $j$ th relay to all the destinations $D_{i}$ with $i=1 \ldots M$ as

$$
C_{j, i}=W \log _{2}\left(1+\gamma_{R_{j}, D_{i}} \beta_{j}\right)
$$

The data rate for cooperative D2D relaying is

$$
C_{j, I}=\min _{i}\left(C_{j, i}\right)
$$


Therefore, by considering the impact of the social trust $\beta_{j}$, the delivery delays are

$$
\begin{aligned}
T_{j} & \simeq \frac{1}{C_{j}} \\
T_{j, I} & \simeq \frac{1}{C_{j, I}}
\end{aligned}
$$

Then, $T_{j, I}$ is the maximum delivery delay related to the worst link to the relay destinations. We consider that the transmit power that needs to transmit in two hops, source-relay and relay-destinations, is not larger than that of the multicast transmission without the use of relays. Regarding the social-trust level $\beta_{j}$, we assume two values, 0 and 1 , where a higher value of $\beta_{j}$ represents a stronger social trust; moreover, we consider that friends have similar behavior in transmitting shared data. For example, a D2D user/relay can choose its friends on its own contact list to define who and how it can help other users for cooperative D2D relaying.

To this end, the optimization problem can be formulated and approximated as

$$
\begin{aligned}
& \min _{j}\left(T_{j}+T_{j, I}\right) \\
& \text { s.t. } \\
& \qquad T_{j}, T_{j, I}>0 \\
& \quad P_{S} T_{j}+P_{R_{j}} T_{j, I}<P_{S_{1}} T_{1}
\end{aligned}
$$

The second constraint (Equation (9)) guarantees that the total transmit power for the two hops due to relay transmission is not larger than that of multicast communication, for which the source transmits with a power $P_{S_{1}}$ directly to the destinations over a total time duration equal to $T_{1}$. The power $P_{S}$ transmitted by the source for the two hops can be different from the power $P_{S_{1}}$.

The optimization problem (Equation (7)) can be solved with a complexity of $O(M * N)$. An exhaustive search to find the optimal solution is affordable, because in the case of multicast communications, the number of participants to the multicast cluster, and consequently the subset of relays, is limited.

In the social relay selection method, we first retrieve the payable transmit power, the geographical locations of each device, and the channel gains and calculate the SNR for each link. Then, we calculate the delivery time needed from the source to each relay and the maximum delivery delay for each group of relay destinations sequentially until the number of relays is reached. We sort the sum related to these delivery times, and, finally, we select the best relay, which assures the minimum delivery time from the source to the destinations. These steps are shown in Algorithm 1 following. 


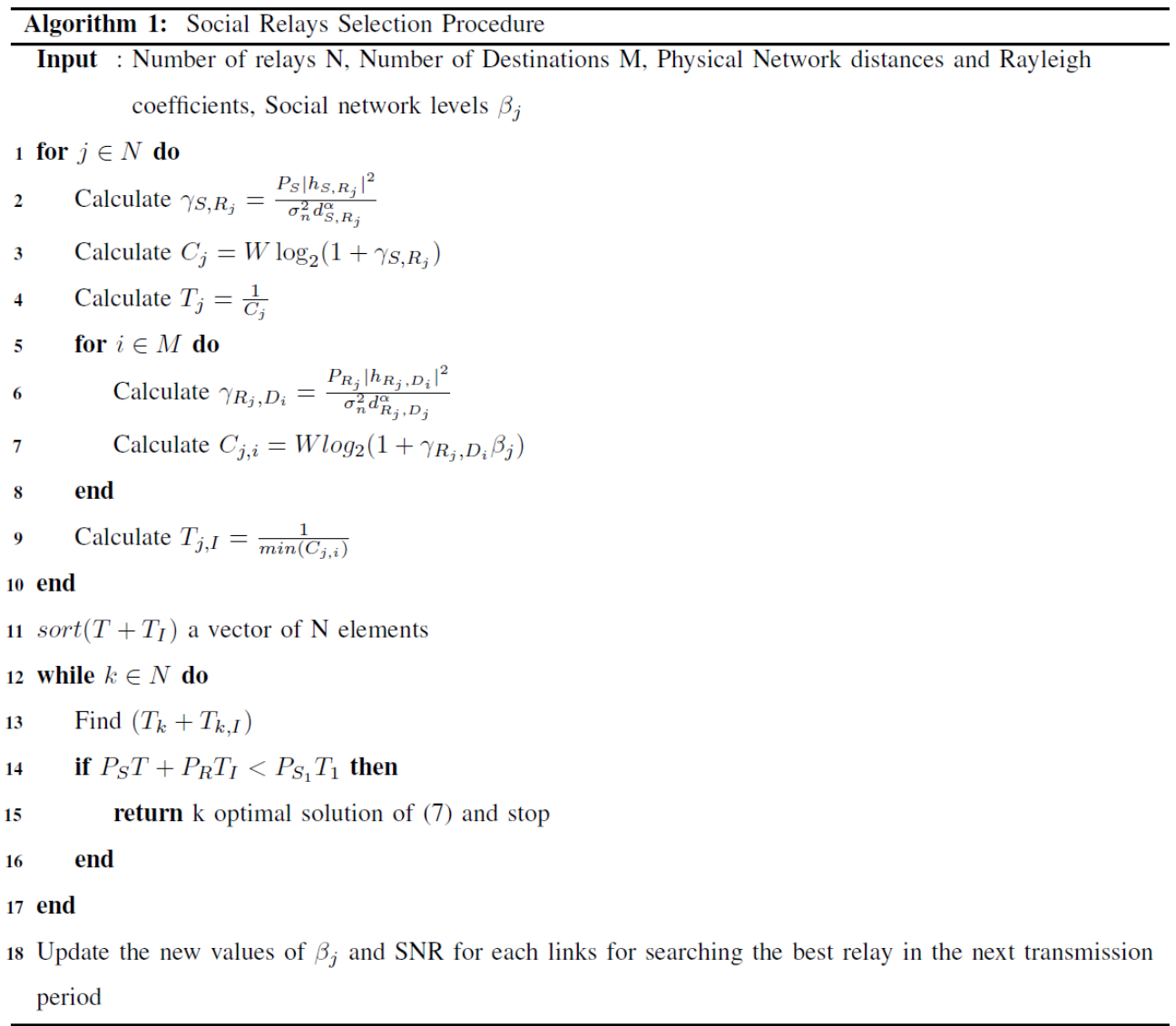

\section{Numerical Results}

In this section, we provide numerical results concerning the performance of the proposed D2D multicast social relaying method. In the considered scenario, a single D2D source device with $R_{j}$ neighbor relay devices, where $j=1 \ldots N$, and $D_{i}$ destination devices, where $i=1 \ldots M$, are located in a single cell with radius $C$, which guarantees a reserved channel for the control communications among these devices. Destination devices are distributed in a restricted area to form a cluster with radius $r$ and $0 \leq r \leq C$. Within the D2D multicast area, the $i$ th device is at the location $\left(d_{i}, \theta_{i}\right)$ with $-r \leq d_{i} \leq r$ and $0 \leq \theta_{i} \leq 2 \pi$, and the distance between two nodes is defined as $d_{j, i}=\sqrt{d_{j}^{2}+d_{i}^{2}-2 d_{j} d_{i} \cos \left(\theta_{j}-\theta_{i}\right)}$. We assume that the distance between the source and the destinations is larger than the distance between any two devices in the multicast group and among the relay and devices and is heavily attenuated because of obstacles, building, and so on. Therefore, the direct link between the source and the destinations does not exist, and communications can be established only via relay.

We consider a transmission channel model often assumed in literature [17], including both the large scale path loss, shadowing variations and zero-mean AWGN. We assume the frequency of non-selective Rayleigh block fading according to a complex Gaussian distribution with zero mean and variance $\sigma_{j, i}^{2}$ for the $i$ th to $j$ th link, that is, constant during one transmission period and changing independently from one period to another. The channel gains $\left|h_{j, i}\right|^{2}$ are exponentially distributed, as in $[10,17]$.

The average SNR for the signal received by a generic D2D destination device from the D2D source device is defined as $\gamma_{S D}$, and the standard deviation is defined as $\sigma_{S d}^{2}$. To simulate an urban environment, we set the $\gamma_{S D}$ value in the region of $0 \mathrm{~dB}$. This choice considers that the direct path has a heavier attenuation, as a result, for example, of obstacles, buildings, and so on, in a generic real multicast D2D communication scenario. The average SNR for the signal received by a D2D destination 
device from one of the D2D relays is defined as $\gamma_{R D}$, and the average SNR for the signal received by a generic $j$ th D2D relay from the D2D source device is $\gamma_{S R}$.

Table 1 shows the values of the main parameters. The value of $\beta_{j}$, as described in the system model, varies depending of the friendship level. If the D2D relay has a friendship connection, it sets its $\beta$ value equal to 1 ; instead, if it has no friendship connection, it reduces the value 10-fold. We suppose that each relay and the D2D source device have the complete knowledge of friendship relations and channel state in terms of the SNR.

Table 1. Main Simulation Parameters.

\begin{tabular}{ll}
\hline Bandwidth & $\mathbf{1 0 ~ M H z}$ \\
\hline Path-loss coefficient & 4 \\
Cell radius & $1 \mathrm{Km}$ \\
Cluster radius & $0.03 \mathrm{Km}$ \\
Number of destinations, $M$ & 10 \\
Number of relays, $N$ & $5: 15$ \\
$\gamma_{S D}$ & $3 \mathrm{~dB}$ \\
$\gamma_{R D}$ & $13-18 \mathrm{~dB}$ \\
Friendship probability & $20 \%$ \\
$\beta_{j}$ for a friend source & 1 \\
$\beta_{j}$ for a non-friend source & 0.1 \\
$\sigma_{S d}$ & $1 \mathrm{~dB}$ \\
$\sigma_{r d}$ & $9 \mathrm{~dB}$ \\
User distribution & $\mathrm{Uniform}$ \\
\hline
\end{tabular}

For a comparative perspective, the throughput normalized with respect to the throughput of direct source-destination transmissions, that is, without the use of relays, is shown in Figure 3 for the number of relays increasing. First, a comparison of the normalized throughput of our method with social and physical information with respect to the stopping approach [18] is shown in the top of Figure 3, for which the direct transmission is added to the source-relay transmission at the receiver, highlighting similar performance.

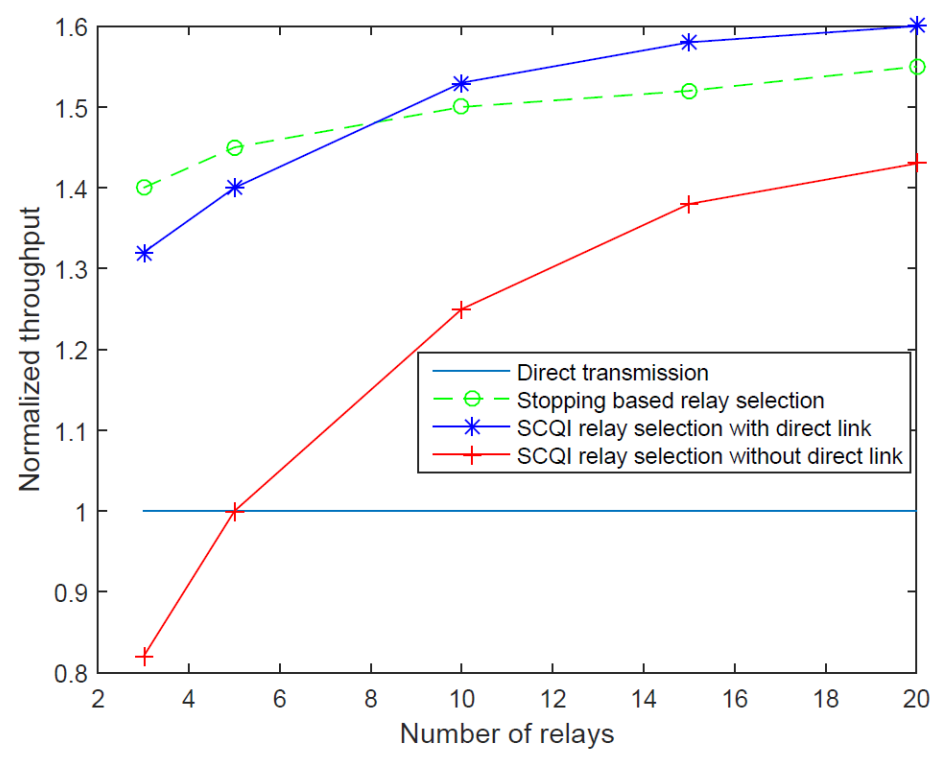

Figure 3. Comparison of normalized throughput with respect to the number of relays for the cases with and without direct transmission. 
If obstacles impact heavily on the transmission, that is, the non-line-of-sight (NLOS) case, the performance degrades, as in Figure 3; this is the more interesting case to analyze because it is closer to real environments. Therefore, in the following, the results refer to the case without direct transmission for the parameter mainly related to the multicast transmission, that is, the end-to-end delivery delay. To emphasize the relevance of social relations in Figure 4, we compare our method, the Social Channel Quality Indicator (SCQI), with two alternatives. In the first, the source selects the relay by taking into account only the friendship level. In particular, it randomly selects a relay from the friends without considering the other neighbor relays. In the second comparison method, the D2D source selects the relay with the minimum delay among the source-relays-destination links only relying on the SNR report. Figure 4 shows the average end-to-end delivery delay for a multicast transmission among the source and all the destinations through the single relay selected, introduced by these three alternatives. To simplify the analysis of the results, the average delays are normalized with respect to the direct-link communication delay obtained for the case that the D2D source can communicate directly with the destinations. Figure 4 shows the importance of the knowledge of both friendship and channel conditions. Our method, which considers both of these, guarantees better performance in terms of transmission delays. Again, the direct link is only shown for reference in this figure.

To evaluate the performance of our method relative to the probability for the relay to be a friend with the source in terms of delivery delay, we consider different friendship probability values, maintaining the same system parameters and channel conditions. The results in Figure 5 show that if the total number of neighbor relays grows, the influence of friendship probability is reduced. Each relay addition improves the delivery delay, as we have a greater probability to find friends that offer their full support in retransmission with the lowest latency in the two-way multicasting.

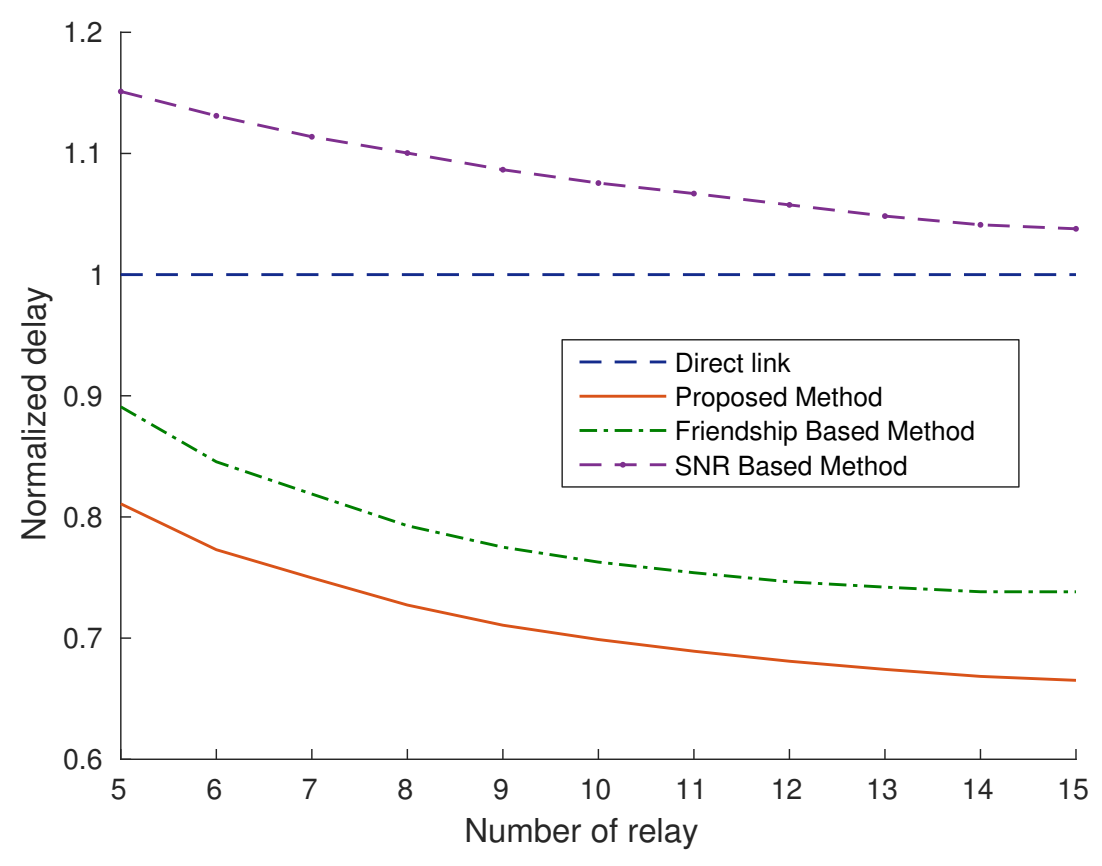

Figure 4. Normalized delay with respect to the number of relays for the considered methods; $\gamma_{R D}=$ $13 \mathrm{~dB}, \gamma_{S D}=3 \mathrm{~dB}, \sigma_{s d}=1 \mathrm{~dB}$, and $\sigma_{r d}=2 \mathrm{~dB}$. 


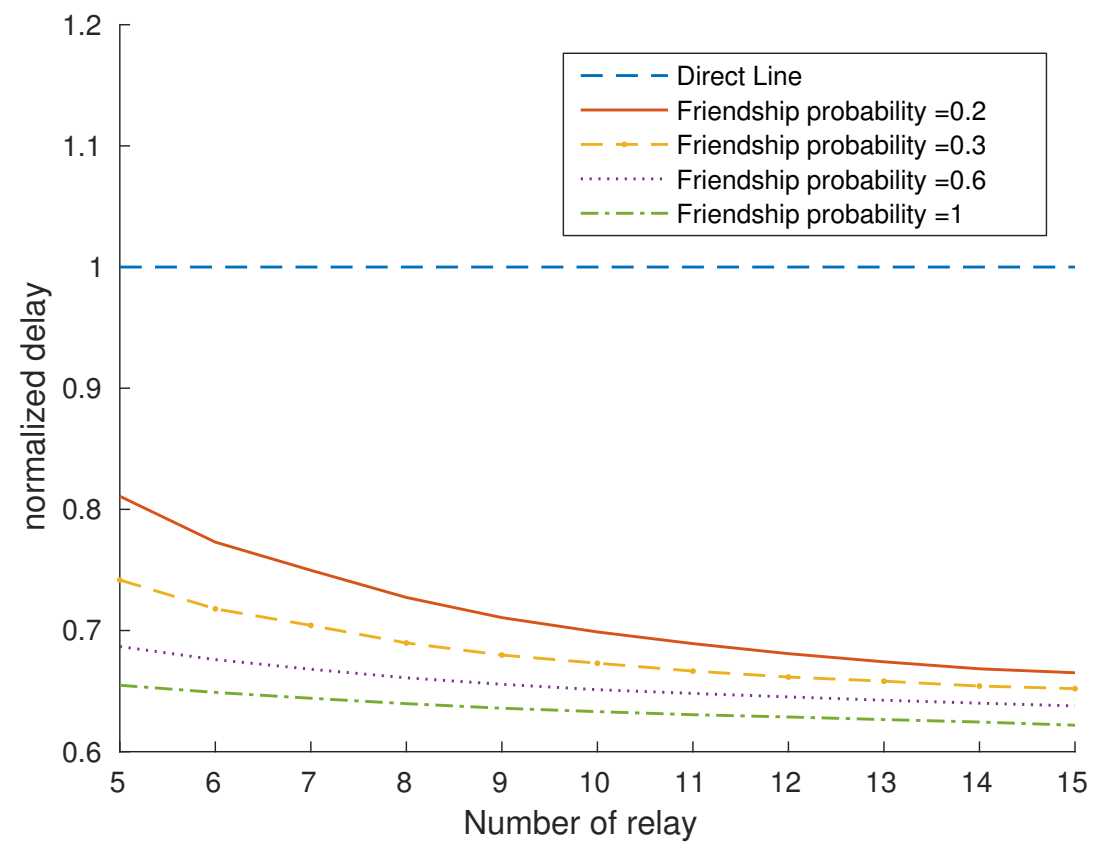

Figure 5. Normalized delay with respect to the number of relays varying the friendship probability; $\gamma_{R D}=13 \mathrm{~dB}, \gamma_{S D}=3 \mathrm{~dB}$, and $\sigma_{s d}=1 \mathrm{~dB}$.

Finally, Figure 6 shows the performance of our method as a function of different $\gamma_{R D}$ values. From this figure, we can note a trend: the normalized delay increases as the $S N R_{r d}$ value decreases and approaches the value of the direct link depending on the number of relays. For example, in the case of $\gamma_{R D}$ equal to $13 d B$, we can note that the direct link is convenient for a number of relays of about seven. This trend is present in each relay-based model: when $\gamma_{S R}, \gamma_{R D}$ and $\gamma_{S D}$ have similar values, the direct communication between the source and destinations becomes the best solution.

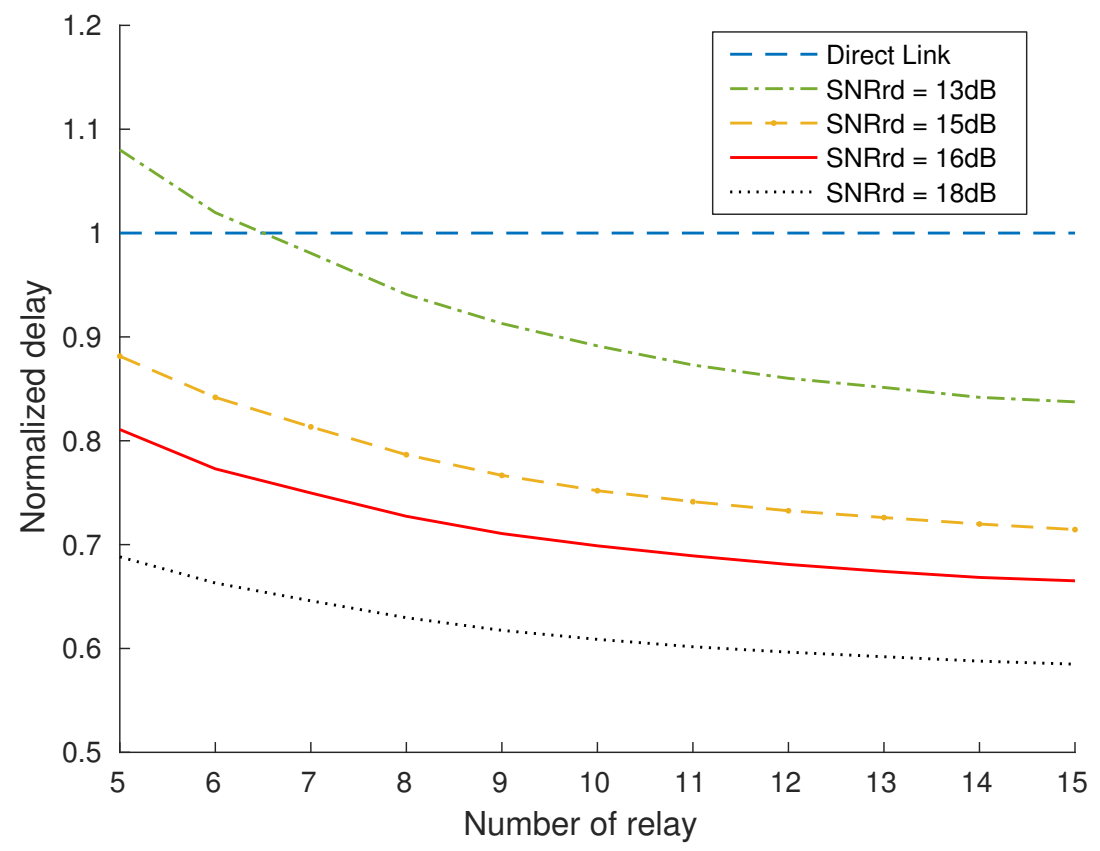

Figure 6. Normalized delay with respect to the number of relays for different $\gamma_{R D}$ values; $\gamma_{S D}=3 \mathrm{~dB}$, and $\sigma_{s d}=1 \mathrm{~dB}$. 


\section{Conclusions}

In this paper, we propose a relay selection method for D2D multicast communication based on D2D channel conditions and social-trust levels. We derive the optimal social-aware relay selection method on the basis of the minimization of the multicast end-to-end delivery time. We further show that our method for social-aware relay selection exhibits an incremental performance with respect to the method that does not use the social domain information, and vice versa for the method in which the relay selection is only based on friendship informations. Numerical results demonstrate that the proposed mechanism can achieve better performance gain when the difference between the channel quality in the two-way source-relay-destinations is better with respect to the source-destinations channel condition.

We have also highlighted the impact of friendship in the decision method. A larger number of friends in the neighborhood achieves a better average performance in terms of transmission delay. Our proposed method can be used in a cell where all the multicast participants are distributed within a small area to reduce the amount of network traffic necessary to deliver digital content to all users belonging to the same social community.

Future developments can be represented by the extension of this method to multi-hop social-aware content distribution, in which each destination is interested in sharing the same digital content with a different community, for example, according to the novel paradigm of fog computing/networking and applications in which clusters of smart devices collaborate toward a common goal.

Furthermore, we can use real-world human mobility traces such as Intel, Infocom06 or Brightkite [25] for future simulations by dividing the devices in the network into several groups to evaluate the system performance in terms of end-to-end delivery delay in realistic environments.

Acknowledgments: This work was partially supported by the project "GAUChO" funded by Progetti di Ricerca di Elevato Interesse Nazionale (PRIN), 2015. The authors thank Dario Di Giacomo for his work to optimize the algorithm.

Author Contributions: All the authors contributed equally to the paper. R. Fantacci coordinated the research activities and supervised the work; F. Chiti and L. Pierucci mainly worked on the system model, designed the experiments and analyzed the simulation results. All the authors wrote the paper and reviewed its structure and intellectual content.

Conflicts of Interest: The authors declare no conflict of interest.

\section{References}

1. Yang, Y.; Hu, H.; Xu, J.; Mao, G. Relay technologies for WiMax and LTE-advanced mobile systems. IEEE Commun. Mag. 2009, 47, 100-105.

2. Nomikos, N.; Skoutas, D.; Makris, P. Relay selection in 5G networks. In Proceedings of the 2014 International Wireless Communications and Mobile Computing Conference (IWCMC), Nicosia, Cyprus, 4-8 August 2014; pp. 821-826.

3. Yu, C.H.; Doppler, K.; Ribeiro, C.B.; Tirkkonen, O. Resource Sharing Optimization for Device-to-Device Communication Underlaying Cellular Networks. IEEE Trans. Wirel. Commun. 2011, 10, 2752-2763.

4. Chiti, F.; Giacomo, D.D.; Fantacci, R.; Pierucci, L. Interference aware approach for D2D communications. In Proceedings of the 2016 IEEE International Conference on Communications (ICC), Kuala Lumpur, Malaysia, 22-27 May 2016; pp. 1-6.

5. Koskela, T.; Hakola, S.; Chen, T.; Lehtomaki, J. Clustering Concept Using Device-To-Device Communication in Cellular System. In Proceedings of the 2010 IEEE Wireless Communication and Networking Conference, Sydney, Australia, 18-21 April 2010; pp. 1-6.

6. Wang, R.; Yang, H.; Wang, H.; Wu, D. Social overlapping community-aware neighbor discovery for D2D communications. IEEE Wirel. Commun. 2016, 23, 28-34.

7. Zhang, Z.; Wang, L.; Zhang, J. Energy Efficiency of D2D Multi-User Cooperation. Sensors 2017, doi:10.3390/s17040697. 
8. Wang, T.; Zhou, Y.; Wang, Y.; Tang, M. Novel Opportunistic Network Routing Based on Social Rank for Device-to-Device Communication. J. Comput. Netw. Commun. 2017, 2017, 2717403.

9. Tannious, R.; Nosratinia, A. Spectrally-efficient relay selection with limited feedback. IEEE J. Sel. Areas Commun. 2008, 26, 1419-1428.

10. Zlatanov, N.; Schober, R. Buffer-Aided Relaying With Adaptive Link Selection-Fixed and Mixed Rate Transmission. IEEE Trans. Inf. Theory 2013, 59, 2816-2840.

11. Simoni, R.; Jamali, V.; Zlatanov, N.; Schober, R.; Pierucci, L.; Fantacci, R. Buffer-Aided Diamond Relay Network with Block Fading. In Proceedings of the IEEE International conference on communications (ICC2015), London, UK, 8-12 June 2015.

12. Simoni, R.; Jamali, V.; Zlatanov, N.; Schober, R.; Pierucci, L.; Fantacci, R. Buffer-Aided Diamond Relay Network With Block Fading and Inter-Relay Interference. IEEE Trans. Wirel. Commun. 2016,15, 7357-7372.

13. Chiti, F.; Fantacci, R.; Pierucci, L. Buffer-aided relaying approaches for multicast communications. In Proceedings of the 2016 International Wireless Communications and Mobile Computing Conference (IWCMC), Paphos, Cyprus, 5-9 September 2016; pp. 411-416.

14. Chiti, F.; Fantacci, R.; Pierucci, L. Dynamic multicast link selections for buffer-aided relaying networks. Int. J. Commun. Syst. 2016, 29, 1790-1804.

15. Chiti, F.; Fantacci, R.; Pierucci, L.; Privitera, N. Optimal joint MIMO and modulation order selection for network coded multicast wireless communications. Telecommun. Syst. 2015, 61, 1-9.

16. Ikhlef, A.; Michalopoulos, D.; Schober, R. Max-Max Relay Selection for Relays with Buffers. IEEE Trans. Wirel. Commun. 2012, 11, 1124-1135.

17. Krikidis, I.; Charalambous, T.; Thompson, J. Buffer-Aided Relay Selection for Cooperative Diversity Systems without Delay Constraints. IEEE Trans. Wirel. Commun. 2012, 11, 1957-1967.

18. Zhang, M.; Chen, X.; Zhang, J. Social-aware relay selection for cooperative networking: An optimal stopping approach. In Proceedings of the 2014 IEEE International Conference on Communications (ICC), Sydney, Australia, 10-14 June 2014; pp. 2257-2262.

19. Zhao, Y.; Li, Y.; Cao, Y.; Jiang, T.; Ge, N. Social-Aware Resource Allocation for Device-to-Device Communications Underlaying Cellular Networks. IEEE Trans. Wirel. Commun. 2015, 14, 6621-6634.

20. Sun, Y.; Wang, T.; Song, L.; Han, Z. Efficient resource allocation for mobile social networks in D2D communication underlaying cellular networks. In Proceedings of the 2014 IEEE International Conference on Communications (ICC), Sydney, Australia, 10-14 June 2014; pp. 2466-2471.

21. Zhang, B.; Li, Y.; Jin, D.; Hui, P.; Han, Z. Social-Aware Peer Discovery for D2D Communications Underlaying Cellular Networks. IEEE Trans. Wirel. Commun. 2015, 14, 2426-2439.

22. Zhang, Y.; Pan, E.; Song, L.; Saad, W.; Dawy, Z.; Han, Z. Social Network Aware Device-to-Device Communication in Wireless Networks. IEEE Trans. Wirel. Commun. 2015, 14, 177-190.

23. Militano, L.; Orsino, A.; Araniti, G.; Iera, A. NB-IoT for D2D-Enhanced Content Uploading with Social Trustworthiness in 5G Systems. Future Internet 2017, 9, doi:10.3390/ fi9030031.

24. Xu, W.; Li, S.; Xu, Y.; Lin, X. Underlaid-D2D-assisted cooperative multicast based on social networks. Peer-to-Peer Netw. Appl. 2016, 9, 923-935.

25. Cho, E.; Myers, S.A.; Leskovec, J. Friendship and Mobility: Friendship and Mobility: User Movement in Location-Based Social Networks. In Proceedings of the 17th ACM SIGKDD International Conference on Knowledge Discovery and Data Mining, San Diego, CA, USA, 21-24 August 2011.

(C) 2017 by the authors. Licensee MDPI, Basel, Switzerland. This article is an open access article distributed under the terms and conditions of the Creative Commons Attribution (CC BY) license (http://creativecommons.org/licenses/by/4.0/). 